\title{
Robotic-assisted thymectomy: current perspectives
}

This article was published in the following Dove Press journal:

Robotic Surgery: Research and Reviews

7 September 2016

Number of times this article has been viewed

\section{Giuseppe Marulli \\ Giovanni M Comacchio \\ Francesca Stocca \\ Davide Zampieri \\ Paola Romanello \\ Francesca Calabrese \\ Alessandro Rebusso \\ Federico Rea}

Thoracic Surgery Unit - Department of Cardiologic, Thoracic and Vascular Sciences, University Hospital,

Padova, Italy
Correspondence: Giuseppe Marulli Thoracic Surgery Unit - Department of Cardiologic, Thoracic and Vascular Sciences, University Hospital, Via Giustiniani 2, 35100, Padova, Italy

Tel +39498218740

Fax +39498212249

Email giuseppe.marulli@unipd.it
Abstract: Thymectomy is the cornerstone in the treatment of thymic tumors and an accepted option for the management of myasthenia gravis. Different surgical approaches have been described, but the gold standard is represented by median sternotomy. In the last two decades, the development of minimally invasive surgery has led to an increased acceptance of thymectomy, especially for benign diseases. Robotic thymectomy seems a further step in the development and evolution of minimally invasive approaches. Since its introduction, different authors described their experience with robotic thymectomy, both for nonthymomatous myasthenia gravis and for thymic tumors. Available data show that robotic thymectomy may be considered a safe and feasible operation. In patients with nonthymomatous myasthenia, robotic thymectomy is effective and the long-term results are encouraging. The role of robotic thymectomy in patients affected by thymoma is still under evaluation, but the intermediate results seem promising both in terms of surgical and oncologic outcomes.

Keywords: thymectomy, thymoma, robot, myasthenia gravis

\section{Introduction}

Thymectomy is one of the most frequent surgical procedures involving the mediastinum, both for benign and malignant diseases.

Since Blalock in the early 20th century demonstrated an improvement of symptoms in a patient with myasthenia gravis (MG) who underwent thymectomy for thymoma and subsequently described the same findings in patients with MG who underwent thymectomy, this operation has played a significant role constituting a widely accepted therapeutic option in the management of nonthymomatous MG. ${ }^{1}$ At the same time, thymectomy is the cornerstone in the treatment of thymic tumors. ${ }^{2}$

A variety of surgical approaches for thymectomy has been described, ranging from open (mainly transternal) to minimally invasive (transcervical or thoracoscopic) approaches, but median sternotomy has been considered the standard approach for many years.

Indeed, the development of minimally invasive surgery has led to an increased acceptance of thymectomy especially for benign diseases, whereas there are still many concerns for thymomas.

The introduction of robotic-assisted surgical systems has been a further step in the development and evolution of minimally invasive approaches with a clear technical advantage over standard video-assisted thoracoscopy, particularly for surgical application in remote-to-reach or narrow anatomical regions, such as the mediastinum. ${ }^{3}$ 
The first surgical application of robotic technique was described by Loulmet et al and Reichenspurner et al in 1999 and was a coronary bypass. ${ }^{4,5}$ In 2001, Yoshino et al described the first robotic operation on the mediastinum, performing a thymectomy for a small thymoma. ${ }^{6}$ Since then, various authors described their experience with robotic thymectomy both for nonthymomatous MG and for thymic tumors. ${ }^{3,7-26}$

Different robotic systems have been developed in the past years but the most widespread is the da Vinci robotic system (Intuitive Surgical, Inc., Sunnyvale, CA, USA).

It consists of an ergonomically designed surgeon's computerized console, a vision system, and a patient-side cart that supports the interactive robotic arms (Figure 1). The surgeon controls the system by sitting at the console far from the patient. The console is connected to the video system and the robotic cart, and it represents the interface between the surgeon and the robotic system. The surgeon sees the operative field through a binocular localized in the upper part of the console and his fingers grasp the master controls below the display and realize the movements of the robotic arms. The system translates the 3D movement of the hands and fingers into precise, identical, and real-time movements of surgical instruments inside the patient's chest.

\section{Surgical technique}

Regarding the surgical technique, the patient is under general anesthesia and has a double-lumen endotracheal tube for selective single lung ventilation during the time of operation. The patient is positioned left- or right-side up (depending on the side of operation based on the surgeon preference) at a $30^{\circ}$ angle with a bean bag (Figure $2 \mathrm{~A}$ ). The operative field should always be prepared and draped for an eventual conversion to median sternotomy. A camera port for the three-dimensional $0^{\circ}$ or $30^{\circ}$ stereo endoscope is introduced through a $15 \mathrm{~mm}$ incision on the fifth intercostal space on the midaxillary line and two additional thoracic ports are inserted: one on the third intercostal space on the midaxillary region and another on the fifth intercostal space on the midclavicular space (Figure 2B). Two arms of the da Vinci system are then attached to the two access points and another arm is attached to the port-inserted endoscope (Figure 3). The left arm has an EndoWrist (Intuitive Surgical, Inc.) instrument that grasps the thymus; the right arm has an Endo-dissector device (Intuitive Surgical, Inc.) with electric cautery function (or a harmonic ultrasound dissector) used to perform the dissection. During surgery, the hemithorax is inflated through the camera port with $\mathrm{CO}_{2}$ ranging in pressure from 6 to $10 \mathrm{mmHg}$. When a left-sided approach is performed (this is our preference), the dissection starts inferiorly at the left pericardiophrenic angle and continues along the anterior border of the phrenic nerve. All anterior mediastinal tissue, including fat, is isolated from

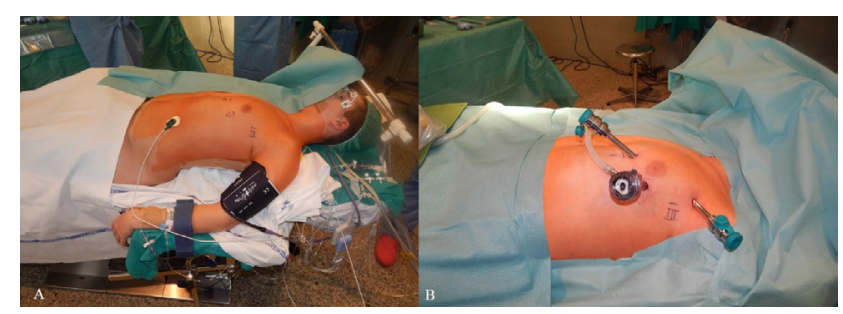

Figure 2 Patient and ports positioning.

Notes: (A) Patient positioned left-side up at a $30^{\circ}$ angle. (B) Ports introduced on the fifth intercostal space on the midaxillary line, fifth intercostal space on the midclavicular space, and third intercostal space on the midaxillary region.

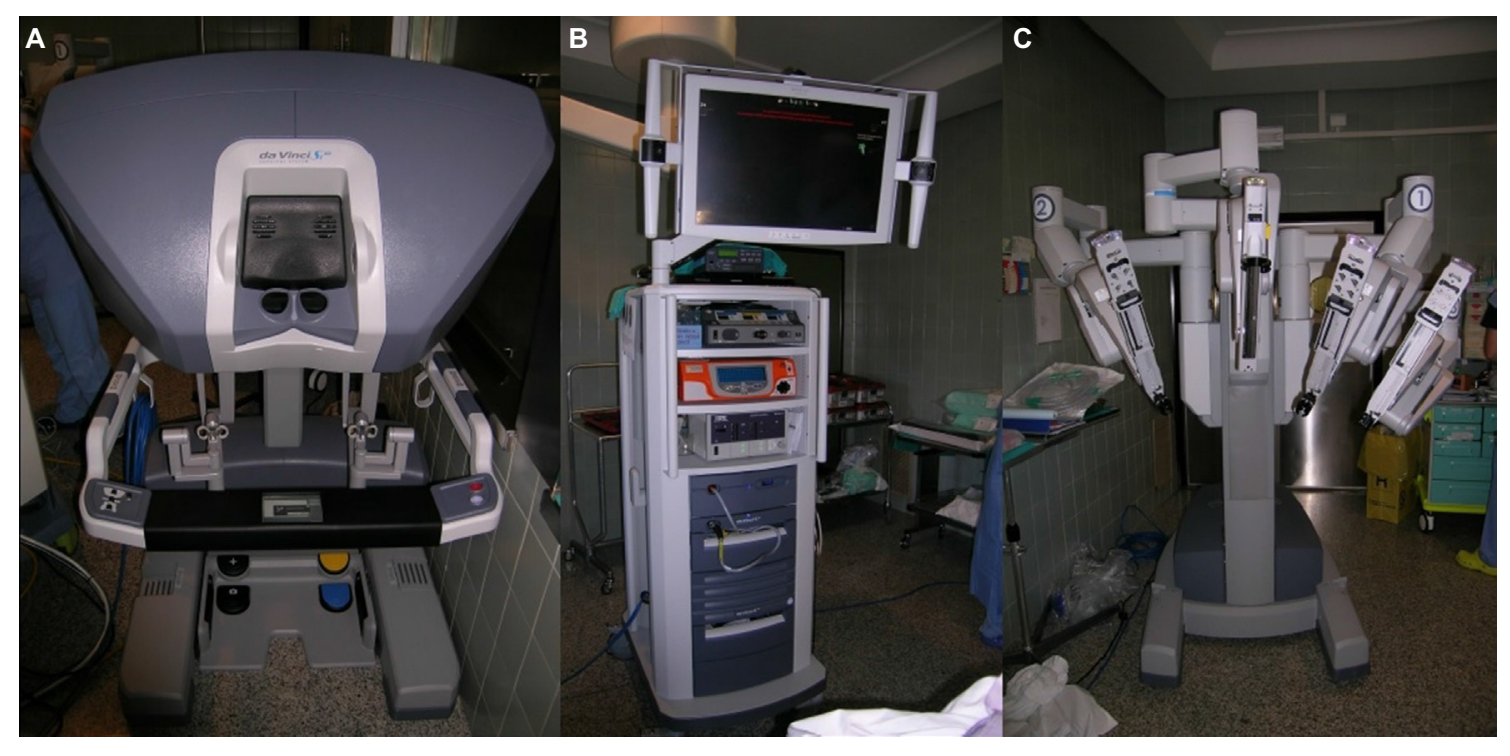

Figure I Robotic components.

Note: (A) surgeon's console, (B) vision system, and (C) patient-side cart with robotic arms. 


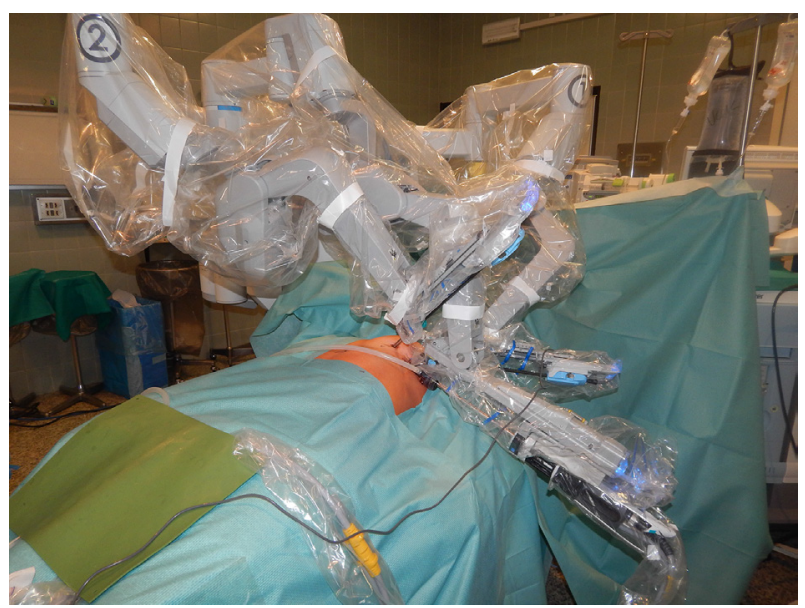

Figure 3 Patient-side cart with robotic arms introduced through the ports.

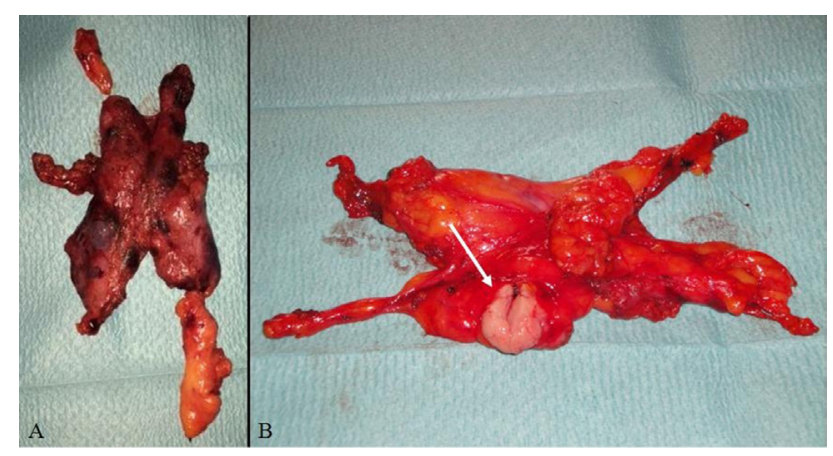

Figure 4 Resected specimens.

Notes: (A) Hyperplastic thymus in patient with MG. (B) Thymus with small thymoma (arrow).

Abbreviation: MG, myasthenia gravis.

the phrenic nerve. The left inferior horn of the thymus is then located and dissected from the pericardium. Subsequently, the thymic gland is separated from the retrosternal area until the right mediastinal pleura and the right inferior horn are found. At this point, the lower part of the thymus is mobilized upward, the left innominate vein is identified, and the dissection continues along the border of the innominate vein up to the point where the thymic veins are identified, clipped, and divided. The dissection continues upward to the neck until the superior horns are identified and divided from the inferior portion of the thyroid gland by a blunt dissection. The thymus gland, anterior mediastinal, and neck fatty tissues are radically resected and the specimen (Figure 4) is placed in an Endobag so that it can be removed through trocar incision. In the right side approach, dissection begins from the cardiophrenic angle and the mediastinal pleura is incised just anterior and medial to the right phrenic nerve, then it continues upward till all anterior mediastinal tissue is separated from the nerve and the superior vena cava. At this point, the dissection follows the retrosternal area by division of the pleura along the lateral border of the right internal mammary vessels from the origin all the way to the diaphragm. The next step involves elevating the thymus off the pericardium, starting from the inferior horns and heading upward until the left brachiocephalic vein is encountered. The thymus is dissected off the anterior aspect of the vein and the thymic veins are identified, clipped, and dissected. Then, the superior horns are identified and divided from the thyroid gland. The left pleura is then opened and after the left phrenic nerve is identified, the dissection of the thymus is completed. After the hemostasis, a $28 \mathrm{~F}$ drainage tube is inserted through the port of the fifth intercostal space, the lung is inflated, and the other wounds are closed. The patient is extubated in the operating room and, after an adequate period of observation, returns to the floor of the surgical thoracic ward. The chest drain is removed if the postoperative chest X-ray shows normal findings and the amount of pleural fluid is permissive, generally 24 hours after operation.

The better side to perform thymectomy is still a matter of debate. The main points that guide the choice of the side include: safety of trocars placement, mediastinal dissection, anatomic considerations over the distribution of the thymic tissue, and surgeon's preference. ${ }^{27}$

Authors supporting the left-sided approach point out that the left lobe of the thymus gland is usually larger and extends down to the pericardiophrenic area, and that the aortopulmonary window and the region below the left innominate vein are frequent sites of ectopic thymic tissue. The thymus may also extend lateral to or under the left phrenic nerve, or descend totally or partially posterior to the innominate vein. ${ }^{27}$ Moreover, the right phrenic nerve is protected by the superior vena cava in the high mediastinum and may be identified and easily followed in the lower part. ${ }^{13,16}$

On the other hand, authors who prefer a right-sided approach describe a larger operative field, a better visualization of the venous confluence by following the superior vena cava, visualization of the aortocaval groove, and a better ergonomic position to accomplish dissection making it easier in the early part of the learning curve. ${ }^{27-29}$

Regardless of the side, it is important that the approach is tailored on the patient's anatomy in order to perform a complete dissection of the thymic tissue, the mediastinal fat from a phrenic nerve to another, and from the superior horns to the cardio-phrenic recess.

Another important point is the surgical technique in case of thymoma: as underlined in the guidelines of International Thymic Malignancy Interest Group, thymectomy for thymoma should be performed following a "no-touch" 
technique..$^{30}$ In this way, the dissection should be accomplished avoiding any manipulation of the tumor in order to allow a safe removal with no risk of capsule breach and dissemination of the tumor in the pleural cavity.

\section{MG and thymectomy}

MG is a disease mediated by autoantibodies (anti-acetylcholine receptor, anti-muscle-specific receptor kinase, and anti-lipoprotein-related protein 4) that also involves the deregulation of helper T-lymphocytes and regulatory T-lymphocytes. This disease is characterized by weakness and skeletal muscle fatigue. ${ }^{31}$

The prevalence of MG is $2.5-10$ per 100,000 persons, and the typical manifestation age is $20-40$ and $60-70$ years. Approximately $10 \%$ of patients with generalized MG do not have detectable serum antibodies against the acetylcholine receptor (seronegative $\mathrm{MG}$ ); a proportion of these patients have serum immunoglobulin $\mathrm{G}$ antibodies against the musclespecific kinase. $^{32}$

Generally, the medical treatment includes corticosteroids, acetylcholine esterase inhibitors, and immune-suppressants. Plasmapheresis and intravenous immunoglobulins are used for myasthenic crisis. For patients with nonthymomatous autoimmune MG, thymectomy is recommended as an option to increase the probability of remission or improvement in symptom control. When surgery is planned, corticosteroids dose should be reduced. In patients with partial symptom control with drugs, preoperative plasmapheresis or intravenous immunoglobulin administration should be considered to reduce the risk of postoperative respiratory failure..$^{33,34}$

There is evidence that thymectomy can be less effective in patients with positive serum titer of antibodies against the muscle-specific receptor tyrosine kinase. ${ }^{35}$

There is no gold standard in surgery timing but it seems that in case of recent onset of symptoms, there is a higher possibility of remission or improvement not depending on symptom severity. ${ }^{34}$ The complete removal of all thymic and mediastinal fatty tissue is associated with higher remission rates. ${ }^{36}$ The major aim of the operative treatment of MG is complete removal of the whole thymic gland but thymectomy is generally not performed in patients over 65 years of age in absence of thymoma or in case of antibody-negative disease. ${ }^{9,37}$

The first thymectomy in patients with MG was performed by Blalock in 1939; since then, thymectomy has been a standard component in the treatment of all stages of MG. ${ }^{38}$

The first thymectomy was performed by median sternotomy and this approach has been the gold standard for many years. In patients with MG, the removal of mediastinal fat is essential so the thymectomy became extended. Zielinski et al demonstrated how extended thymectomy performed by sternotomy produces a major complete remission of $\mathrm{MG}$ in a higher percentage of patients. The removal of mediastinal fat which contains foci to ectopic thymus is essential for remission or improvement of this neurological disease. ${ }^{39}$

In 1995, Yim et al first performed thoracoscopic thymectomy in eight patients with MG: they obtained a decrease in postoperative pain and hospitalization compared with patients who underwent thymectomy by sternotomy. ${ }^{40}$

Between 1995 and 2008, Pompeo et al performed 32 thoracoscopic thymectomy for MG but in two cases it was necessary to convert to median sternotomy because of technical difficulties in dissecting the thymus in one patient and diffuse uncontrolled bleeding in the other. ${ }^{35}$ Since then, several other authors reported good experiences in thoracoscopic thymectomy for MG, emphasizing the good results both in terms of surgical and perioperative outcome (short hospitalization, less pain, low complication rate, and good cosmetics) and long-term neurological results (remission or improvement of symptoms in $>90 \%$ of patients).$^{41}$ Despite these promising results, the thoracoscopic approach did not gain a wide diffusion for some reasons: 1) the thymectomy is not extended being difficult to have a complete dissection of the fatty tissue, particularly in the neck and contralateral mediastinum; 2) the thoracoscopic thymectomy is considered technically difficult with an estimated learning curve of at least 50 cases; and 3) the procedure is considered less safe compared to transternal approach with regard to the risk of intraoperative vascular and nervous injuries.

The first robotic thymectomy for MG was described by Ashton et al and Rea et al in 2003: Ashton et al used a right-sided approach with completion of the operation through the leftsided approach, while Rea used a left-sided approach only. ${ }^{42,43}$

Since these first case reports, many authors supported the robotic thymectomy for MG treatment reporting outstanding results both in terms of surgical and neurological outcomes (Table 1). In particular, the improvement of symptoms rates ranged between $77 \%$ and $>90 \%$, with complete remission rates reaching values between $16.2 \%$ and $>40 \%$, comparable with those reported for open approach.

\section{Thymoma and thymectomy}

Thymomas are rare tumors, representing $0.2 \%-1.5 \%$ of all malignancies, but are the most common tumors of the anterior mediastinum, accounting for $50 \%$ of all anterior mediastinal tumors. ${ }^{44}$ Surgery is the mainstay in the treatment of thymomas and radical resection represents the 
most important prognostic factor. ${ }^{45,46}$ Surgery is generally performed through a median sternotomy, which is considered the standard approach, allowing a technically easy and oncologically safe operation. ${ }^{47}$

Although in the last decades minimally invasive surgery has greatly developed in different oncological fields, several surgeons have been reluctant to use these surgical approaches for early stage thymoma. ${ }^{48}$

The main concerns are represented by the possible rupture of the capsule with implantation of the tumor during endoscopic manipulations and the supposed increased risk of local recurrence (due to reduced safety margins after minimally invasive resection). ${ }^{49,50}$ Furthermore, the lack of long-term oncologic results, learning curve required to perform this operation safely, and relative rarity of these tumors are additional reasons that slow down the spread of the thoracoscopic/ robotic resection for early stage thymomas.

However, two reports comparing oncologic outcome between thoracoscopic and open resection of thymoma showed no significant differences in intermediate-term follow-up..$^{51,52}$

Recently, Friedant et al performed a systematic review and meta-analysis of the current literature regarding minimally invasive versus open thymectomy for thymic malignancies. Data showed no significant differences in $\mathrm{R} 0$ resection rate and locoregional recurrence in patients with Masaoka stage I and II tumors in the two groups, whereas there was a significant lesser blood loss and postoperative stay in the minimally invasive group. ${ }^{50}$

In the last years, some authors have described their experience in robotic thymomectomy for thymoma focusing on the oncologic outcome (Table 1). These series are generally small, single-center, retrospective experiences. However, available data confirm that this approach is technically sound and safe in the hands of appropriately trained surgeons: no major complications and no mortality occurred in these series, with only a mean of $6.6 \%$ of open conversion.

Regarding the oncologic point of view, these reports show a low recurrence rate of the tumor with only four $(2.1 \%)$ patients experiencing recurrence (one case was a thymic carcinoma, one a Masaoka stage IV thymoma, whereas in the other two cases the stage of the primary disease was not specified). However, the majority of these series are characterized by a low number of cases and limited followup, making available data promising but still inconclusive regarding the oncologic outcome. Thymomas are generally indolent tumors with long recurrence time, especially in early stages. Indeed, a long lapse of time (at least 10 years) is necessary to evaluate the actual survival and relapse rate.
In the published studies, the longest follow-up has a mean time of 51.7 months. ${ }^{16}$

As pointed out by Davenport, there is a lack of evidence in current literature supporting a minimally invasive approach compared to standard sternotomy. ${ }^{46}$ Further studies with longer follow-up are necessary to confirm the real oncologic outcome of robotic technique.

Cheng et al proposed some radiological criteria to better select candidates for minimally invasive thymoma resection: the location in the anterior mediastinum, tumor encapsulation, a distinct fat plane between the tumor and vital organs, existence of residual normal appearing thymic tissue, no mass compression effect, and unilateral tumor predominance, particularly for tumors of dimension $>3 \mathrm{~cm}$, are the main characteristics supporting a minimally invasive approach (Figure 5). ${ }^{53}$

Regarding the appropriate size of thymoma for robotic resection, it is still a matter of debate. The majority of the literature deals with lesions smaller than $5 \mathrm{~cm}$, but an average size tumor around $3 \mathrm{~cm}$ is generally considered as oncologically acceptable. ${ }^{16,48} \mathrm{~A}$ large tumor size may be not considered an absolute contraindication; however, it may interfere with the thoracoscopic procedure making the manipulation more difficult, with an increased chance of an open conversion or it may prolong the operative time. ${ }^{16}$

Recently, the International Thymic Malignancy Interest Group proposed some standard policies regarding minimally invasive resection of thymomas. The tumor should be removed en bloc with the surrounding thymus and fatty tissue, according to surgical oncologic principles, in order to reduce the risk of recurrence also for capsulated thymomas. For other than Masaoka stage I tumors, transcapsular invasion can be subtle and may not be detected by the surgeon, and therefore safety margins are necessary. ${ }^{30}$ Frequently, clinical Masaoka stage I thymomas are found to be stage II at pathological examination, with microscopical or rather macroscopical invasion of the surrounding tissue already evident at surgery. Marulli et al in a European series of robotic thymectomy described $62 \%$ of Masaoka stage II thymomas, despite most cases being clinically diagnosed as stage $\mathrm{I}^{16}$ Similarly, Takeo et al found $57 \%$ of patients with Masaoka stage II and III after an initial diagnosis of stage I, whereas Quintanilla-Martinez et al reported that $28.5 \%$ of tumors described as encapsulated showed a microscopic capsular invasion. ${ }^{54,55}$ Considering all series of robotic thymectomy for thymoma reported to date, $2.5 \%$ of patients were found to have a Masaoka stage III disease.

Moreover, thymomas should be resected using a no-touch technique; it should not be grasped or squeezed with retractors because of possible rupture of the capsule and concern 


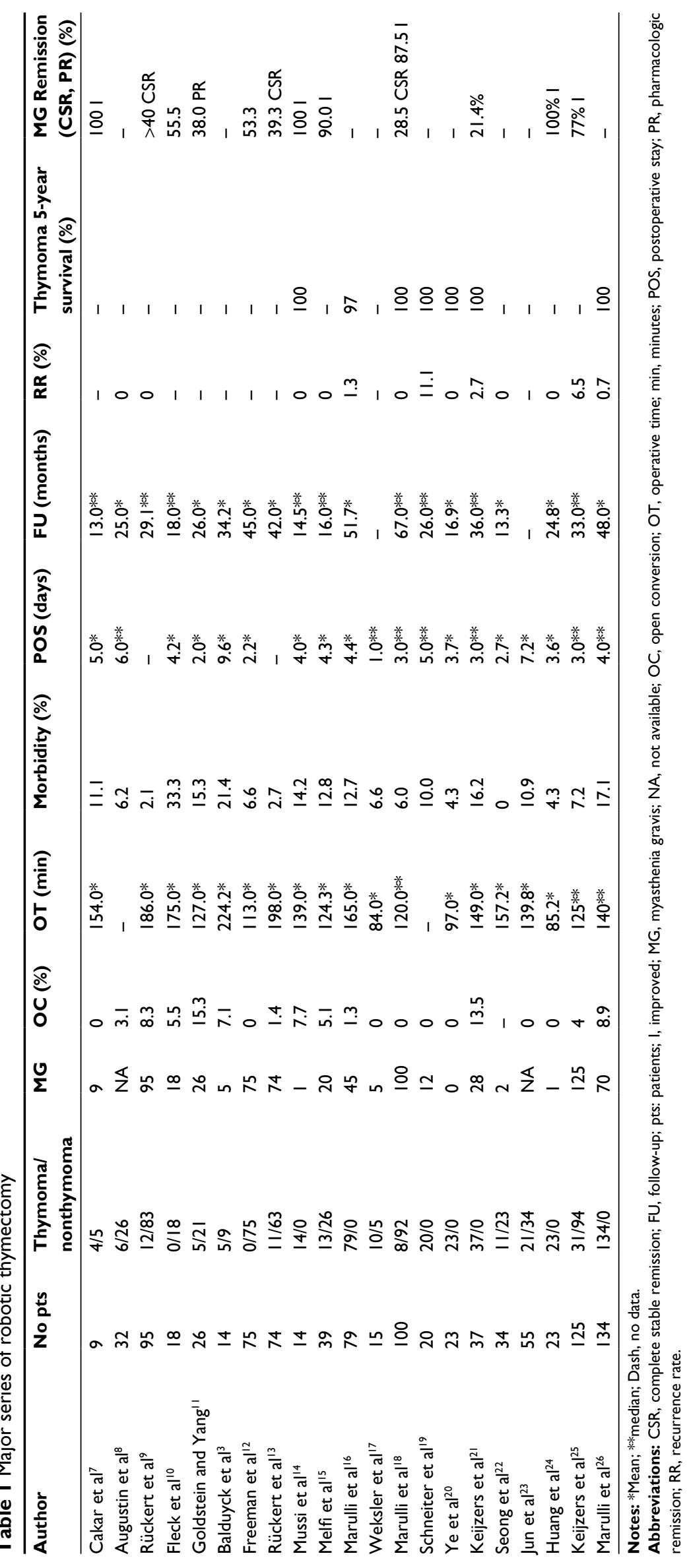




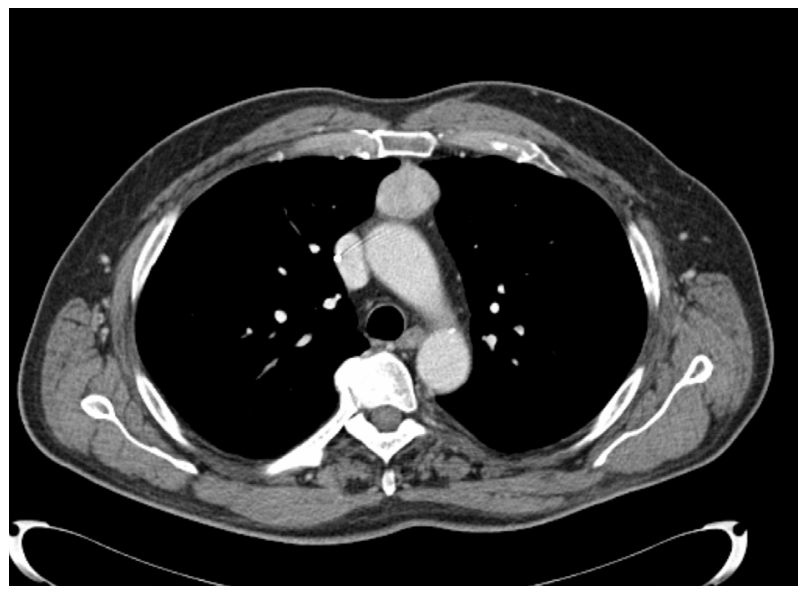

Figure 5 Chest CT scan (mediastinal window) showing small middle-mediastinal thymoma.

Abbreviation: CT, computed tomography.

of an increased risk of pleural dissemination. ${ }^{30}$ The no-touch technique requires a more complex and accurate dissection, and thus a longer learning curve and longer operative time. ${ }^{16}$

Certainly, if an initial attempt of minimally invasive approach is deemed by the surgeon to be unlikely to be completed, both for the risks of the procedure itself or possible violation of any principle of oncologic safety, open conversion is mandatory. Interestingly, conversion rate in the published series is only $6.2 \%$, suggesting an appropriate selection of the patients.

\section{Transternal versus robotic thymectomy}

To date, median sternotomy is considered the gold standard for thymectomy. In literature, there are several studies comparing transsternal with thoracoscopic thymectomy, but only few authors have compared transsternal with robotic thymectomy (Table 2) ${ }^{37,17,20,22}$ Cakar et al published the first series comparing robotic versus transsternal thymectomy, showing a better clinical outcome with a lower rate of complications and reduced postoperative stay in the robotic group.? However, the small number of patients and nonrandomized retrospective design make it very difficult to generalize these results. Balduyck et al compared the two approaches using quality of life assessment questionnaires showing a better postoperative recovery in patients who underwent robotic surgery. ${ }^{3}$ Other authors corroborated the previous findings, describing a reduced blood loss and hospital stay in robotic series compared to transsternal. ${ }^{17,20,22}$ Median sternotomy shows some specific complications, related to the surgical access itself, as sternal instability, wound healing impairment, wound infection, and hematomas. The use of steroids

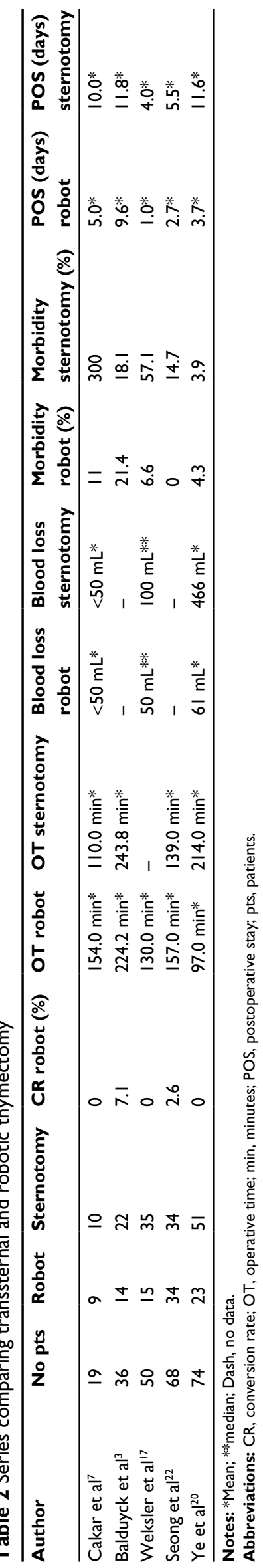


in MG patients who undergo sternotomy has also raised controversies because of the supposed increased risk in wound complications ${ }^{56}$ Robotic thymectomy avoids complications of median sternotomy, and all the studies showed less postoperative complications (Table 2), although in just one this finding was statistically significative. ${ }^{17}$ Indeed, the lower morbidity of minimally invasive techniques, particularly robotic, has led to a greater acceptance of these operation, especially for benign diseases, with earlier referral of myasthenic patients to the surgeon.

\section{Transcervical and thoracoscopic versus robotic thymectomy}

Different minimally invasive surgical approaches have been described for thymectomy, historically the most common represented by the transcervical and the thoracoscopic approaches.

In some centers, cervicotomy represents a common minimally invasive approach for thymectomy in patients with MG and small thymomas. ${ }^{57}$

This surgical access has important advantages over sternotomy in terms of early referral to surgery, preservation of lung function, low rate of morbidity, reduced postoperative pain, and hospital stay. In patients with $\mathrm{MG}$, remission rate seems to be similar or slightly lower to those after transsternal thymectomy. ${ }^{58}$

Regarding thymomas, poor data are available. As mentioned before, resection of thymomas through minimally invasive techniques is not generally accepted by all surgeons, especially in the suspicion of invasiveness. Thymomas resected through cervicotomy are small size lesions or occasional findings in patients who underwent surgery for MG, and thus no specific follow-up data are generally available. One of the major series in literature has been described by Maggi et al with 110 noninvasive thymomas treated through transcervical access, sometimes widened by a limited split of sternum manubrium, with four patients having a local relapse of thymoma. ${ }^{57}$

This technique shows some disadvantages: the standard approach described by Cooper provides the neck hyperextension and the sternum retraction anteriorly, which could lead to increased postoperative pain. ${ }^{59}$ It could be also difficult in elderly patients with poor neck extension or in obese patients. ${ }^{60}$ Moreover, dissection may not be always easily completed through a simple cervical approach, requiring sometimes modification of the technique, as sternal split or videothoracoscopic assistance. ${ }^{61,62}$
To date, there are no studies in literature comparing transcervical to robotic thymectomy and the only possible comparison is based on crude data reported in the single studies. Whereas cervicotomy enables a complete dissection and removal of the upper horns of the gland, it may be challenging to obtain an extensive thymectomy and removal of all the mediastinal fat through the single cervical incision, thus requiring an additional thoracoscopic aid or a partial sternotomy. ${ }^{57,59}$ Robotic approach, through its superior view of the operating field, permits a complete dissection of the thymus also in difficult-to-reach areas of the mediastinum. On the other hand, cervicotomy avoids chest drains and could have thus lower postoperative pain and shorter hospital stay. ${ }^{60}$

The robotic approach for thymectomy may be considered as an evolution of standard thoracoscopy, but to date only three authors performed a real comparison between these two technical options regarding the surgical results (Table 3). In 2011, Rückert et al performed a retrospective analysis of the results of 74 consecutive robotic thymectomies compared with 79 thoracoscopic thymectomies. No significant differences were found in terms of conversion rate, operative time, and postoperative complications, but there was an improved outcome for myasthenic patients after robotic thymectomy. ${ }^{13}$ Ye et al found no significant differences in a cohort of 21 robotic and 25 thoracoscopic thymectomies for early stage thymoma, except for a reduced postoperative stay in the former group, which showed anyway higher hospitalization costs. ${ }^{49}$ Jun et al described only a longer operative time for the robotic thymectomy, considering the setup and the console time. ${ }^{23}$ All authors, however, pointed out that robotic surgery is a feasible and safe technique showing surgical advantages when compared with standard thoracoscopy.

Augustin et al compared their institutional experience on robotic thymectomy with the literature on standard thoracoscopy describing a similar postoperative stay, but shorter operative time for robotic approach with higher hospitalization costs. ${ }^{8}$ The thoracoscopic approach showed a wide range of operative time, probably caused by the learning curve, different amount, and frequency of this type of operation among various institutions and differences in patients and disease enrolled in the published studies.

All studies so far did not clearly favor any minimally invasive technique and no prospective randomized trials comparing outcomes are available. Therefore, only general statements are made about the preference toward the robotic approach, based on impressions and personal feeling. It is undoubted that the robotic approach for thymectomy has all the same advantages of standard minimally invasive 
approaches, such as reduced postoperative pain, blood loss, operative time, patient morbidity and mortality, infection rate and postoperative hospital stay, preserved pulmonary function, and good cosmetic results. Moreover, it seems to overcome the disadvantages of standard thoracoscopy: the two-dimensional view of the operating field, the surgeon's tremor enhanced by the long thoracoscopic instruments, and the fact that these instruments do not articulate make it difficult to operate in a tiny, delicate, and difficult-to-reach space with large and vulnerable nerves and vessels as the mediastinum. On the contrary, the improved dexterity of robotic instruments that can articulate with $7^{\circ}$ of freedom and rotate $360^{\circ}$ allows complex three-dimensional movements and the filtering of hand tremor enhances a safe dissection around vessels and nerves. The high resolution, three-dimensional video image of the robot permits the best possible and magnified view of the surgical field. ${ }^{1,16}$ These advantages seem to minimize difficulties, particularly in cases of challenging thymectomy, such as in obese patients (body mass index $>35$ ), patients who underwent previous sternotomy, or with atypical thymus gland or pectus excavatum. ${ }^{63}$

Moreover, the surgeon operates in an ergonomic and comfortable position, whereas $87 \%$ of surgeons report experience of some kind of pain related to performing minimally invasive procedures. ${ }^{17}$

Regarding learning curves, thoracoscopic thymectomy has always been considered a technically challenging operation, with a long learning curve. ${ }^{48}$ Jun et al showed a decrease in operative time in their robotic series, with significant results comparing the first eleven cases to the subsequent eleven and a substantial reduction of nearly 30 minutes in the latest 20 cases. Compared to thoracoscopic technique, there was a slight difference in operative time, but the authors pointed out that the comparison was made between the first 55 cases of the robotic series and the last 60 of 300 cases of thoracoscopic thymectomy. ${ }^{23}$ Hence, the authors state that robotic surgery has a shorter learning curve and is easier to manipulate. Other authors have confirmed the shorter learning curve of robotic thymectomy ${ }^{13,15,63}$ Anyway, robotic thymectomy is frequently performed in centers that already perform thoracoscopic thymectomy; therefore, it should be taken into consideration that this experience may have paved the way for the robotic thymectomy.

Some major disadvantages have been described regarding robotic surgery. First, the high cost of this type of operation. Augustin et al evaluated that the use of the robot is significantly more expensive than conventional thoracoscopy 
with an additional cost of $\sim 91 \%$, primarily caused by the initial capital costs of the robotic system, annual maintenance, and disposable materials. While the former may be reduced by the multidisciplinary management of the robotic system, the latter are due to the expensive robotic instruments, which can be reused only a limited number of times. ${ }^{8,15}$ High cost may be partially compensated by a shorter hospital stay and fewer complications or redo-operations, but no clear data are available to date. ${ }^{17}$

Some authors pointed out the lack of tactile feedback that could theoretically increase the risk of damaging delicate structures. However, this disadvantage seems to be widely compensated by the superior view of the operating field through the three-dimensional vision. ${ }^{16,17}$

Another disadvantage concerns the placement of the surgeon, away from the patient and operating at a nonsterile console..$^{9,17}$ Thus, another surgeon able to perform an emergency conversion needs to stay sterile near the robot. However, the undocking of the robotic system could make emergency conversion more difficult.

\section{Conclusion}

In conclusion, robotic thymectomy may be considered a safe and feasible operation. Thymectomy in patients with nonthymomatous myasthenia is effective and the long-term results are encouraging. The role of robotic thymectomy in patients affected by thymoma is still under evaluation with the intermediate results being very good both in terms of surgical and oncologic outcomes.

\section{Disclosure}

The authors report no conflicts of interest in this work.

\section{References}

1. Rea F, Marulli G, Bortolotti L, Feltracco P, Zuin A, Sartori F. Experience with the "daVinci" robotic system for thymectomy in patients with myasthenia gravis: report of 33 cases. Ann Thorac Surg. 2006;81(2):455-459.

2. Detterbeck FC, Zeeshan A. Thymoma: current diagnosis and treatment. Chin Med J (Engl). 2013;126(11):2186-2191.

3. Balduyck B, Hendriks JM, Lauwers P, Mercelis R, Ten Broecke P, Van Schil P. Quality of life after anterior mediastinal mass resection: a prospective study comparing open with robotic-assisted thoracoscopic resection. Eur J Cardiothorac Surg. 2011;39(4):543-548.

4. Loulmet D, Carpentier A, d'Attellis N, et al. Endoscopic coronary artery bypass grafting with the aid of robotic assisted instruments. $J$ Thorac Cardiovasc Surg. 1999;118(1):4-10.

5. Reichenspurner H, Damiano RJ, Mack M, et al. Use of the voicecontrolled and computer-assisted surgical system ZEUS for endoscopic coronary artery bypass grafting. J Thorac Cardiovasc Surg. 1999; 118(1):11-16.

6. Yoshino I, Hashizume M, Shimada M, Tomikawa M, Tomiyasu M, Suemitsu R, Sugimachi K. Thoracoscopic thymomectomy with the da Vinci computer-enhanced surgical system. J Thorac Cardiovasc Surg. 2001; 122(4):783-785.
7. Cakar F, Werner P, Augustin F, Schmid T, Wolf-Magele A, Sieb M, Bodner J. A comparison of outcomes after robotic open extended thymectomy for myasthenia gravis. Eur J Cardiothorac Surg. 2007;31(3): 501-504.

8. Augustin F, Schmid T, Sieb M, Lucciarini P, Bodner J. Video-assisted thoracoscopic surgery versus robotic-assisted thoracoscopic surgery thymectomy. Ann Thorac Surg. 2008;85(2):S768-S771.

9. Rückert JC, Ismail M, Swierzy M, et al. Thoracoscopic thymectomy with the da Vinci robotic system for myasthenia gravis. Ann N Y Acad Sci. 2008;1132:329-335.

10. Fleck T, Fleck M, Muller M, Hager H, Klepetko W, Wolner E, Wisser W. Extended videoscopic robotic thymectomy with the da Vinci telemanipulator for the treatment of myasthemia gravis: the Vienna experience. Interact Cardiovasc Thorac Surg. 2009;9(5):784-787.

11. Goldstein SD, Yang SC. Assessment of robotic thymectomy using the Myasthenia Gravis Foundation of America Guidelines. Ann Thorac Surg. 2010;89(4):1080-1086.

12. Freeman RK, Ascioti AJ, Van Woerkom JM, Vyverberg A, Robison RJ. Long-term follow up after robotic thymectomy for nonthymomatous myasthenia gravis. Ann Thorac Surg. 2011;92(3):1018-1023.

13. Rückert JC, Swierzy M, Ismail M. Comparison of robotic and nonrobotic thoracoscopic thymectomy: a cohort study. J Thorac Cardiovasc Surg. 2011;141(3):673-677.

14. Mussi A, Fanucchi O, Davini F, Lucchi M, Picchi A, Ambrogi MC, Melfi F. Robotic extended thymectomy for early-stage thymomas. Eur J Cardiothorac Surg. 2012;41(4):e43-e46.

15. Melfi F, Fanucchi O, Davini F, Viti A, Lucchi M, Ambrogi MC, Mussi A. Ten-year experience of mediastinal robotic surgery in a single referral centre. Eur J Cardiothorac Surg. 2012;41(4):847-851.

16. Marulli G, Rea F, Melfi F, et al. Robot-aided thoracoscopic thymectomy for early-stage thymoma: a multicenter European study. JThorac Cardiovasc Surg. 2012;144(5):1125-1130.

17. Weksler B, Tavares J, Newhook TE, Greenleaf CE, Diehl JT. Robotassisted thymectomy is superior to transsternal thymectomy. Surg Endosc. 2012;26(1):261-266.

18. Marulli G, Schiavon M, Perissinotto E, Bugana A, Di Chiara F, Rebusso A, Rea F. Surgical and neurological outcomes after robotic thymectomy in 100 consecutive patients with myasthenia gravis. JThorac Cardiovasc Surg. 2013;145(3):730-736.

19. Schneiter D, Tomaszek S, Kestenholz P, Hillinger S, Opitz I, Inci I, Weder W. Minimally invasive resection of thymomas with the da Vinci ${ }^{\circledR}$ surgical system. Eur J Cardiothorac Surg. 2013;43(2):288-292.

20. Ye B, Li W, Ge XX, et al. Surgical treatment of early-stage thymomas: robot-assisted thoracoscopic surgery versus transsternal thymectomy. Surg Endosc. 2014;28(1):122-126.

21. Keijzers M, Dingemans AM, Blaauwgeers H, et al. 8 years' experience with robotic thymectomy for thymomas. Surg Endosc. 2014;28(4): 1202-1208.

22. Seong YW, Kang CH, Choi JW, Kim HS, Jeon JH, Park IK, Kim YT. Early clinical outcomes of robot-assisted surgery for anterior mediastinal mass: its superiority over a conventional sternotomy approach evaluated by propensity score matching. Eur J Cardiothorac Surg. 2014;45(3): e68-e73.

23. JunY, Hao L, Demin L, Guohua D, Hua J, Yi S. Da Vinci robot-assisted system for thymectomy: experience of 55 patients in China. Int JMed Robot. 2014;10(3):294-299.

24. Huang P, Ye B, Yang Y, Tantai JC, Zhao H. Experience with the "da Vinci" robotic system for early-stage thymomas: report of 23 cases. Thorac Cancer. 2014;5(4):325-329.

25. Keijzers M, de Baets M, Hochstenbag M, et al. Robotic thymectomy in patients with myasthenia gravis: neurological and surgical outcomes. Eur J Cardiothorac Surg. 2015;48(1):40-45.

26. Marulli G, Maessen J, MelfiF, et al. Multi-institutional European experience of robotic thymectomy for thymoma. Ann Cardiothorac Surg. 2016;5(1): $18-25$.

27. Kumar A, Asaf BB. Robotic thoracic surgery: the state of the art. $J$ Minim Access Surg. 2015;11(1):60-67. 
28. Deen S, Farivar AS, Louie BE. Thoracic techniques: robotic thymectomy for thymoma. Indian J Surg Oncol. 2013;4(2):132-137.

29. Nakamura H, TaniguchiY. Robot-assisted thoracoscopic surgery: current status and prospects. Gen Thorac Cardiovasc Surg. 2013;61(3):127-132.

30. Toker A, Sonett J, Zielinski M, Rea F, Tomulescu V, Detterbeck FC. Standard terms, definitions, and policies for minimally invasive resection of thymoma. J Thorac Oncol. 2011;6(7 Suppl 3):S1739-S1742.

31. Jakubíková M, Pit'ha J, Marečková H, Týblová M, Nováková I, Schutzner J. Two-year outcome of thymectomy with or without immunosuppressive treatment in nonthymomatous myasthenia gravis and its effect on regulatory T cells. J Neurol Sci. 2015;358(1-2):101-106.

32. Evoli A, Tonali PA, Padua L, et al. Clinical correlates with anti-MuSK antibodies in generalized seronegative myasthenia gravis. Brain. 2003; 126(10):2304-2311.

33. Sardenberg RA, Abadalla RZ, Abreu IR, Evaristo EF, Younes RN. Robotic thymectomy for myasthenia gravis. J Bras Pneumol. 2011;37(5): 694-696.

34. Mineo TC, Pompeo E, Lerut TE, Bernardi G, Coosemans W, Nofroni I. Thoracoscopic thymectomy in autoimmune myasthenia: results of left-sided approach. Ann Thorac Surg. 2000;69(5):1537-1541.

35. Pompeo E, Tacconi F, Massa R, Mineo D, Nahmias S, Mineo TC. Longterm outcome of thoracoscopic extended thymectomy for nonthymomatous myasthenia gravis. Eur J Cardiothorac Surg. 2009;36(1):164-169.

36. Jurado J, Javidfar J, NewmarkA, et al. Minimally invasive thymectomy and open thymectomy: outcome analysis of 263 patients. Ann Thorac Surg. 2012;94(3):974-982.

37. Alkhawajah NM, Oger J. Treatment of myasthemia gravis in the aged. Drugs Aging. 2015;32(9):689-697.

38. Meyer DM, Herbert MA, Sobhani NC, et al. Comparative clinical outcomes of thymectomy for myasthenia gravis performed by extended transsternal and minimally invasive approaches. Ann Thorac Surg. 2009; 87(2):385-391.

39. Zielinski M, Kuzdzal J, Szlubowski A, Soja J. Comparison of late results of basic transsternal and extended transsternal thymectomies in the treatment of myasthenia gravis. Ann Thorac Surg. 2004;78(1):253-258.

40. Yim AP, Kay RL, Ho JK. Video-assisted thoracoscopic thymectomy for myasthenia gravis. Chest. 1995;108(5):1440-1443.

41. Zahid I, Sharif S, Routledge T, Scarci M. Video-assisted thoracoscopic surgery or transsternal thymectomy in the treatment of myasthenia gravis? Interact Cardiovasc Thorac Surg. 2011;12(1):40-46.

42. Ashton RC Jr, McGinnis KM, Connery CP, Swistel DG, Ewing DR, De Rose JJ Jr. Totally endoscopic robotic thymectomy for myasthenia gravis. Ann Thorac Surg. 2003;75(2):569-571.

43. Rea F, Bortolotti L, Girardi R, Sartori F. Thoracoscopic thymectomy with the 'da Vinci' surgical system in patient with myasthenia gravis. Interact Cardiovasc Thorac Surg. 2003;2(1):70-72.

44. Srirajaskanthan R, Toubanakis C, Dusmet M, Caplin ME. A review of thymic tumours. Lung Cancer. 2008;60(1):4-13.

45. Regnard JF, Magdeleinat P, DromerC, DulmetE, de MontprevilleV, Levi JF, Levasseur P. Prognostic factors and long-term results after thymoma resection: a series of 307 patients. J Thorac Cardiovasc Surg. 1996; 112(2):376-384
46. Davenport E, Malthaner RA. The role of surgery in the management of thymoma: a systematic review. Ann Thorac Surg. 2008;86(2):673-684.

47. Masaoka A, Monden Y, Nakahara K, Tanioka T. Follow-up study of thymomas with special reference to their clinical stages. Cancer. 1981; 48(11):2485-2492.

48. Toker A, Erus S, Ozkan B, Ziyade S, Tanju S. Does a relationship exist between the number of thoracoscopic thymectomies performed and the learning curve for thoracoscopic resection of thymoma in patients with myasthenia gravis? Interact Cardiovasc Thorac Surg. 2011;12(2):152-155.

49. Ye B, Tantai JC, Li W, Ge XX, Feng J, Cheng M, Zhao H. Video-assisted thoracoscopic surgery versus robotic-assisted thoracoscopic surgery in the surgical treatment of Masaoka stage I thymoma. World J Surg Oncol. 2013;11:157.

50. Friedant AJ, Handorf EA, Su S, Scott WJ. Minimally invasive versus open thymectomy for thymic malignancies: systematic review and meta-analysis. J Thorac Oncol. 2015;11(1):30-38.

51. Pennathur A, Qureshi I, Schuchert MJ, et al. Comparison of surgical techniques for early-stage thymoma: feasibility of minimally invasive thymectomy and comparison with open resection. JThorac Cardiovasc Surg. 2011;141(3):694-701.

52. Cheng YJ, Kao EL, Chou SH. Videothoracoscopic resection of stage II thymoma: prospective comparison of the results between thoracoscopy and open methods. Chest. 2005;128(4):3010-3012.

53. Cheng YJ, Hsu JS, Kao EL. Characteristics of thymoma successfully resected by videothoracoscopic surgery. Surg Today. 2007;37(3):192-196.

54. Takeo S, Tsukamoto S, Kawano D, Katsura M. Outcome of an original video-assisted thoracoscopic extended thymectomy for thymoma. Ann Thorac Surg. 2011;92(6):2000-2005.

55. Quintanilla-Martinez L, Wilkins EW Jr, Ferry JA, Harris NL. Thymomamorphologic subclassification correlates with invasiveness and immunohistologic features: a study of 122 cases. Hum Pathol. 1993;24(9):958-969.

56. Zieliński M, Kuzdzał J, Staniec B, et al. Safety for preoperative use of steroids for transsternal thymectomy in myasthenia gravis. Eur $J$ Cardiothorac Surg. 2004;26(2):407-411.

57. Maggi G, Giaccone G, Donadio M, et al. Thymomas. A review of 169 cases, with particular reference to results of surgical treatment. Cancer. 1986;58(3):765-776.

58. de Perrot M, Bril V, McRae K, Keshavjee S. Impact of minimally invasive trans-cervical thymectomy on outcome in patients with myasthenia gravis. Eur J Cardiothorac Surg. 2003;24(5):677-683.

59. Ampollini L, Del Rio P, Sianesi M, Rusca M, Carbognani P. Transcervical video-assisted thymectomy: preliminary results of a modified surgical approach. Langenbecks Arch Surg. 2011;396(2):267-271.

60. Shrager JB. Extended transcervical thymectomy: the ultimate minimally invasive approach. Ann Thorac Surg. 2010;89(6):S2128-S2134.

61. Shrager JB, Nathan D, Brinster CJ, Yousuf O, Spence A, Chen Z, Kaiser LR. Outcomes after 151 extended transcervical thymectomies for myasthenia gravis. Ann Thorac Surg. 2006;82(5):1863-1869.

62. Bramis J, Diamantis T, Tsigris C, et al. Video-assisted transcervical thymectomy. Surg Endosc. 2004;18(10):1535-1538.

63. Ismail M, Swierzy M, Rückert JC. State of the art of robotic thymectomy. World J Surg. 2013;37(12):2740-2746.
Robotic Surgery: Research and Reviews

\section{Publish your work in this journal}

Robotic Surgery: Research and Reviews is an international, peer reviewed, open access, online journal publishing original research, commentaries, reports, and reviews on the theory, use and application of robotics in surgical interventions. Articles on the use of supervisory-controlled robotic systems, telesurgical devices, and shared-control systems are
Dovepress

invited. The manuscript management system is completely online and includes a very quick and fair peer review system, which is all easy to use. Visit http://www.dovepress.com/testimonials.php to read real quotes from published authors. 